\title{
Upregulation of Limk1 caused by microRNA-138 loss aggravates the metastasis of ovarian cancer by activation of Limk1/cofilin signaling
}

\author{
PUXIANG CHEN, MENGJUN ZENG, YAN ZHAO and XIAOLIN FANG \\ Department of Gynecology and Obstetrics, The Second Xiangya Hospital of \\ Central South University, Changsha, Hunan 410011, P.R. China
}

Received April 30, 2014; Accepted June 24, 2014

DOI: $10.3892 /$ or.2014.3461

\begin{abstract}
LIM kinase 1 (Limk1) is associated with cell proliferation and metastasis and its dysregulated expression has been observed in many types of cancer. The present study aimed to examine the role of Limk1 in the development of ovarian cancer, as well as the underlying molecular mechanism involved. The results showed that increased Limk1 and decreased miR-138 expression co-existed in ovarian cancer. Furthermore, knockout of Limk1 or the overexpression of miR-138 resulted in reduced cell invasion and migration, while silencing of miR-138 led to enhancement of the invasion and migration of ovarian cancer cells. Cell growth was inhibited by the overexpression of miR-138, although not by the knockout of Limk1. miR-138 directly targeted Limk1 and inhibited ovarian cancer cell growth by PCNA and Bcl-2. Moreover, Limk1/cofilin/p-cofilin is likely a critical signaling pathway involving in miR-138 modulation of ovarian cancer cell metastasis. The results provide evidence supporting miR-138/Limk1 as a novel diagnostic or therapeutic target for ovarian cancer.
\end{abstract}

\section{Introduction}

Ovarian cancer (OC) is one of the most sensitive solid tumors which have a high mortality rate, failure rate and few treatment options (1). Although significant advances in the detection and effort to reduce recurrence rates have been made, the 5-year survival rate has remained relatively unchanged for over 20 years $(2,3)$. The main obstacle of OC therapy is recurrence, which is characterized by metastasis and chemoresistance. The primary aim of therapy remains palliation and control

Correspondence to: Professor Xiaolin Fang, Department of Gynecology and Obstetrics, The Second Xiangya Hospital of Central South University, 139 Middle Renmin Road, Changsha, Hunan 410011, P.R. China

E-mail: csufangxiaolin@163.com

Key words: ovarian cancer, LIM kinase 1, miR-138, metastasis, cofilin, Talen technology of symptoms for patients with recurrent OC (3). Thus, the identification of key molecular mediators for metastasis and chemoresistance in OC is essential.

LIM kinase 1 (Limk1) is a member of a novel class of serine-threonine protein kinases, playing an important role in reorganization of actin cytoskeleton through inactivating phosphorylation of the ADF family protein cofilin, which depolymerizes actin filaments. Maintenance of the actin dynamics in an ordered manner is essential for stabilization of the cell shape or promotion of cell motility depending on the cell type. These are the two key phenomena that may become altered during acquisition of the metastatic phenotype by cancer cells (4). A high expression of Limk1 has been detected in various invasive cancers. In 2003, Davila et al found that Limk1 was associated with the proliferation and invasiveness of prostate cancer cells (4). The important role of Limk1 in mediating migration and invasion was subsequently verified in colorectal cancer cells (5), breast cancer cells $(6)$, and non-small cell lung cancer $(7,8)$. Zhang et al (9) found a significant correlation between increasing Limk1 protein levels and increasing disease severity/poor levels of differentiation of ovarian carcinoma, suggesting that Limk1 is involved in mechanisms regulating cell invasion and may be involved in ovarian carcinoma progression. However, the functional role of Limk1 and the related molecular mechanism in OC remain unclear.

MicroRNAs (miRNAs) can regulate gene expression by inhibiting gene translation or facilitating mRNA degradation. Dysregulation of miRNAs is a potential molecular mechanism of the abnormal expression of oncogenes and tumor-suppressor genes (10). MicroRNA-138 (miR-138) is a classical anti-oncomiR that, can inhibit the growth of non-small cell lung cancer through the downregulation of EZH2 (11), suppress invasion and promote apoptosis in head and neck squamous cell carcinoma $(12,13)$, decrease epithelial-mesenchymal transition in squamous cell carcinoma cell lines (14), and induce cell cycle arrest by targeting cyclin D3 in hepatocellular carcinoma (15). miR-138 downregulation maintains $\mathrm{NF}-\kappa \mathrm{B}$ activation and promotes lipid raft formation in esophageal squamous cell carcinoma (16), promotes colorectal cancer metastasis by targeting TWIST2 (17), and increases the proliferation, migration and invasion of cholangiocarcinoma cells through the 
upregulation of RhoC/p-ERK/MMP-2/MMP-9 (18). Although miR-138 is able to suppress the invasion and metastasis of OC cells by targeting SOX4 and HIF-1 $\alpha$ (19), whether other critical molecules such as Limk are involved in actions of miR-138 in OC requires confirmation, as Limk is predicted as a direct target of miR-138 by available bioinformatics software.

In the present study, the effects of Limk1 and miR-138 on the migration and invasion of $\mathrm{OC}$ cells and their regulatory inter-relationship were investigated. The data suggest that the the downregulation of miR-138 induced the upregulation of Limk1 in OC cells. Thus, miR-138/Limk1 may play a key role in the migration and invasion of OC cells.

\section{Materials and methods}

Cell culture. OC cell lines SKOV3, HOC1, H0-8910, OVAC and the IOSE386 normal ovarian epithelial cell line were purchased from the China Center for Type Culture Collection (CCTCC; Wuhan, China) and maintained in RPMI-1640 medium (HyClone) containing 10\% fetal bovine serum (FBS; Invitrogen Life Technologies, Grand Island, NY, USA) in a humidified atmosphere of $5 \% \mathrm{CO}_{2}$ at $37^{\circ} \mathrm{C}$.

Tissue microarray immunohistochemistry. The present study was approved by the Ethics Committee of the Central South University, Changsha, China. The tissue microarray (TC0217; Auragene, Nanjing China) covering 80 case OC samples of different $\mathrm{TNM}$ stages $(\mathrm{T} 1=15, \mathrm{~T} 2=10, \mathrm{~T} 3=25, \mathrm{~T} 4=15$ and $\mathrm{TNF}$ undefined=5) and 10 normal ovarian tissues were processed for immunohistochemistry. Tissue microarray blocks were incubated with anti-Limk1 antibody (Abcam, Cambridge, MA, USA) or normal rabbit IgG as the negative control. Immunostaining was performed using the Moticam 3000 System with diaminobenzidine (Zhongshan Jinqiao Co., Beijing, China). Image-Pro Plus software was used to quantify the mean density of Limk1 staining, according to the manufacturer's instructions.

Quantitative RT-PCR. Total RNA was extracted from the cells with TRIzol (Invitrogen Life Technologies). The relative expression level of miR-138 was determined by quantitative RT-PCR (RT-qPCR) using a mirVana ${ }^{\mathrm{TM}}$ real-time RT-PCR microRNA detection kit (Ambion, Austin, TX, USA) following the manufacturer's instructions. Specific primer sets for miR-138 and U6 (internal reference) were obtained from GeneCopoeia, Guangzhou, China. The expression of Limk1 mRNA was detected by RT-qPCR using the standard SYBRGreen RT-PCR kit (Takara, Shiga, Japan) following the manufacturer's instructions. The specific primer pairs used were: Limk1 , sense, 5'-ACCTCAACTCCCACAACT-3' and antisense, 5'-TCTCGCACAGGACGATCC-3'; with $\beta$-actin as an internal control, sense, 5'-AGGGGCCGGACTCGTCAT ACT-3' and antisense, 5'-GGCGGCACCACCATGTACCCT-3'. The relative expression of Limk1 mRNA or miR-138 was quantified using the GraphPad Prism 4.0 software (GraphPad Software, San Diego, CA, USA) and the $2^{-\Delta \Delta \mathrm{Ct}}$ method.

Western blotting. Cells were solubilized in cold RIPA lysis buffer. After that, proteins were separated with $8 \%$ SDS-PAGE, and then transferred to a PVDF membrane. The membranes were blocked in 5\% non-fat dried milk in PBST for $3 \mathrm{~h}$ and then incubated overnight with relative specific primary antibodies (Abzoom, Dallas, TX, USA) with $\beta$-actin as a control. After incubation with the appropriate secondary antibody, immune complexes were detected using an ECL kit (Auragene Bioscience Inc., Changsha, China).

Luciferase reporter assay. The normal and mutated 3'-UTR of Limk1 were constructed by PCR, and then inserted into the MCS in the psiCHECK ${ }^{\mathrm{TM}}-2$ vector (Promega, Madison, WI, USA). For the luciferase reporter assay, $2 \times 10^{4}$ SKOV3 cells were cultured to $\sim 50-60 \%$ confluence in a 24 -well plate. For each well, the medium was replaced by $300 \mu$ I OPTI-MEM medium (Invitrogen, Carlsbad, CA, USA). The cells were co-transfected with psiCHECK ${ }^{\mathrm{TM}}-2$ Limk1-3'-UTR or psiCHECK ${ }^{\mathrm{TM}}$-2-Limk1-mut 3'-UTR vector plus $50 \mathrm{nM}$ miR-138 mimics or $100 \mathrm{mM}$ miR-138 inhibitor using Cellfectin ${ }^{\circledR}$ II reagent (Invitrogen Life Technologies), according to the manufacturer's instructions. The cells were incubated with transfection reagent/DNA complex for $5 \mathrm{~h}$, and then refreshed with fresh complete medium. A dual-luciferase reporter assay system (Promega) was used to determine the luciferase activities $48 \mathrm{~h}$ after co-transfection. Renilla luciferase activity was normalized to firefly luciferase activity.

Cell proliferation assay. An MTT assay was used to measure cell proliferation. Cells in exponential growth were plated at a final concentration of 2,000 cells/well in 96-well plates. Cell viability was evaluated by MTT assay after 24, 48, 72 and $96 \mathrm{~h}$ of seeding. The optical density at $570 \mathrm{~nm}$ (OD570) of each well was measured with an ELISA reader (ELx800 type; BioTek Instruments, Winooski, VT, USA). The experiment was repeated three times.

Cell invasion assay. The invasive ability of OC cells was studied in 24-well Transwell chambers (Chemicon International, Temecula, CA, USA), which has a layer of Matrigel. For each group, $200 \mu \mathrm{l}$ of cell suspension $\left(1 \times 10^{6}\right.$ cells $\left./ \mathrm{ml}\right)$ was added in triplicate wells. After 24-h incubation, the number of cells that had migrated through the filter into the lower wells was determined by gentian violet assay.

Cell migration assay. Cell migratory ability was estimated using a wound healing assay. In brief, cells were cultured to a confluence of ?. Wounds of $\sim 1 \mathrm{~mm}$ width were created with a plastic scriber, and the cells were washed and incubated in a serum-free medium. After wounding for $24 \mathrm{~h}$, the cells were incubated in a medium including $10 \%$ fetal bovine serum. Cultures at 0, 24 and $48 \mathrm{~h}$ were fixed and observed under a microscope.

Talen construction and cell transfection. To generate Limk1 or cofilin knockout stable cell lines, Limk1- and cofilin-Talen plasmid pair were constructed. A Talen kit was used to knock out the Limk1 or cofilin genes in SKOV3 and HO-8910 cells according to the manufacturer's instructions. Briefly, the plasmids pCMV-NLS-N-terminal-L1-C-terminal-Fok1(L)IRES-PURO-pA and pCMV-NLS-N-terminal-R1-Cterminal-Fok1(R)-pA were against the Talen recognition module from the 5'- and 3'-ends. The Talen sequences of 


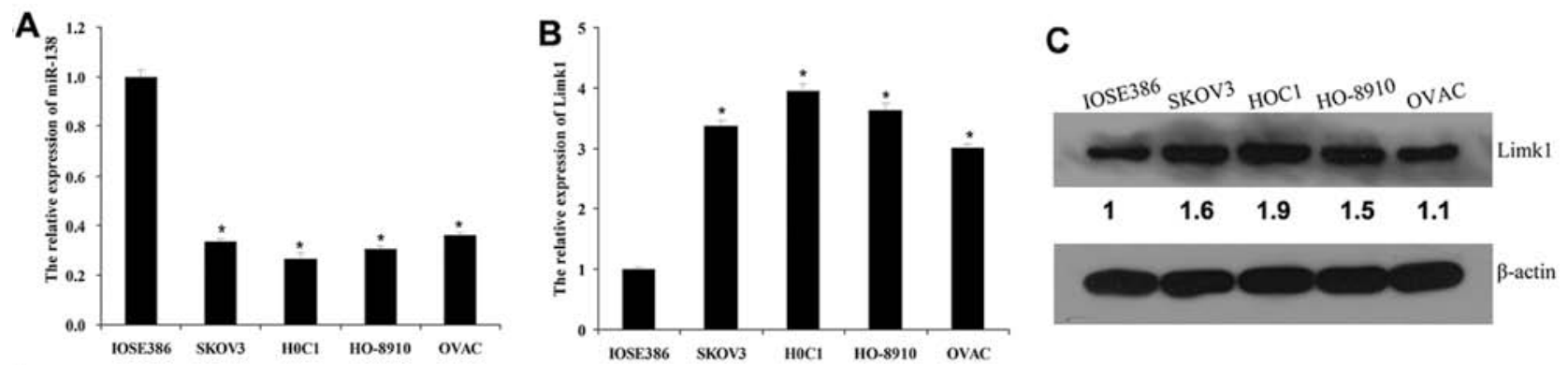

D
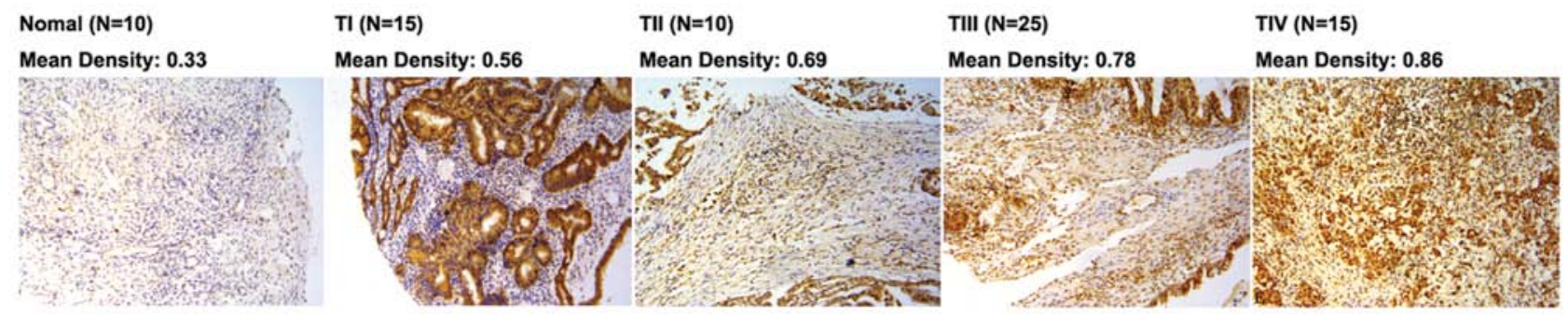

Figure 1. Expression of Limk1 and miR-138 in OC. (A) Relative expression of Limk1 mRNA in human OC cell lines compared to the IOSE386 normal ovarian cell line (Con) using RT-qPCR. (B) Relative expression of miR-138 mRNA in human OC cell lines compared to the IOSE386 normal ovarian cell line (Con) using RT-qPCR. (C) Relative expression of Limk1 protein in human OC cell lines compared to the IOSE386 normal ovarian cell line (Con) using western blot analysis. The numbers listed under the lanes are the relative quantification of each band. (D) The representative results of Limk1 expression (brown particles) in human OC tissue compared to normal, by immunohistochemical analysis. " $\mathrm{P}<0.05$ vs. Con, in SKOV3 and HO-8910 cells.

Limk1 were: left arm, GACGCTACTTTGTTGCACC and right arm, CGCTTCCTTCCTCTCCCA. The Talen sequences of cofilin were: left arm, GACGCTACTTTGTTGCACC and right arm, GAAGACTTACGCACCT. Sequencing was then performed to determine the sequences of the two plasmids. Three sequencing primers were used as follows: 5'-CTCCCC TTCAGCTGGACAC-3'; 5'-AGCTGGGCCACGATTGAC-3'; 5'-GGGAGCACCCCTCAACCTGAC-3'. After confirming that the sequences of these two plasmid pairs were correct, the two pairs of plasmids were co-transfected into SKOV3 and HO-8910 cells using Lipofectamine 2000 (Invitrogen, Life Technologies), respectively, according to the manufacturer's instructions. The mRNA level of Limk1 and cofilin was subsequently assessed to confirm the efficiency of Talen-mediated Limk1 or cofilin KO using RT-qPCR. For the miR-138 functional analysis, the cells were transfected with the pre- or anti-miR-138 to generate gain-of-function or loss-of-function cell models, respectively. Transfection was performed according to the manufacturer's instructions.

Statistical methods. Statistical analysis was performed by SPSS 17 software. The results are expressed as the mean \pm SD of three independent experiments. For each experiment, the statistical analysis was repeated three times. Statistical analysis of differences was performed by one-way analysis of variance (ANOVA) or two-way ANOVA. The null hypothesis was rejected at the 0.01 level.

\section{Results}

Expression of miR-138 and Limk1 in OC cells and tissues. RT-qPCR was performed to detect the miR-138 and Limk1 mRNA levels in the IOSE386 normal ovarian epithelial cell line and SKOV3, HOC1, HO-8910 and OVAC OC cell lines.
The relative expression of miR-138 was significantly lower in the four OC cell lines compared to the control IOSE386 cells. By contrast, the levels of Limk1 mRNAs significantly increased in OC cell lines as compared to the control (Fig. 1A and B). Additionally, the Limk1 protein was confirmed to be higher in OC cells as compared to the control (Fig. 1C). The results suggested a negative relationship between miR-138 and Limk1 in OC cells. To evaluate the correlation between Limk1 expression and the progression of $\mathrm{OC}$, immunohistochemical analysis of tissue microarrays was performed. The expression of Limk1 in OC tissues was clearly increased compared to normal ovarian tissues. Furthermore, the expression of Limk1 was gradually elevated with the malignant progression of OC (Fig. 1D). This suggested that increased Limk1 may contribute to the malignant progression of $\mathrm{OC}$.

Limkl is a novel target of miR-138 in SKOV3 and HO-8910 cells. A luciferase reporter assay was performed to determine whether Limk1 was a direct target of miR-138 in OC SKOV3 and HO-8910 cells, based on the bioinformatic prediction that the binding site for miR-138 at the 3'-UTR of Limk1 mRNA was strong and conserved. Data of the luciferase reporter assay showed that the activity of luciferase was significantly decreased only after transfection with 3'-UTR of the Limk1 gene in the miR-138 treated cells. However, in the other groups, the activity of luciferase showed no difference (Fig. 2A and B). These findings suggested that Limk1 is a direct target of miR-138 in OC cells.

miR-138 negatively regulates the protein expression of Limk1 in SKOV3 and HO-8910 cells. The effects of miR-138 overexpression or downregulation on the mRNA level of Limk1 in SKOV3 and HO-8910 cells were investigated. Overexpression of miR-138 significantly inhibited the mRNA level of Limk1 
A

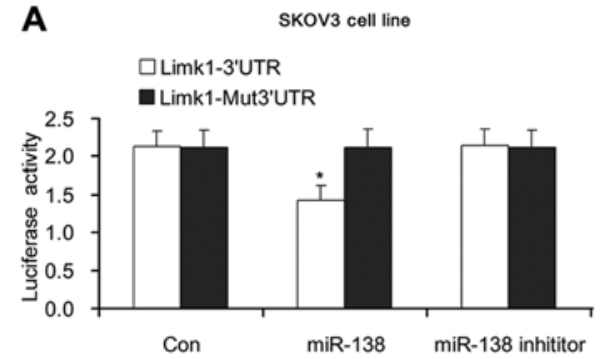

B

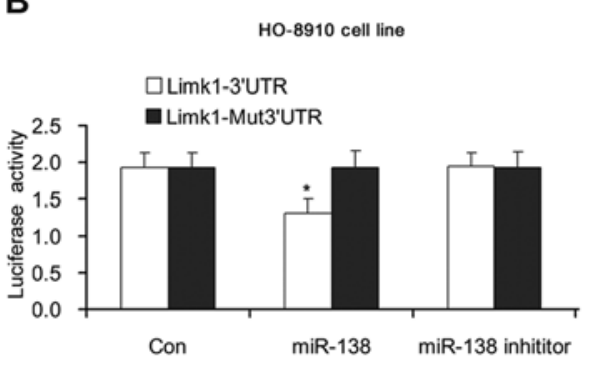

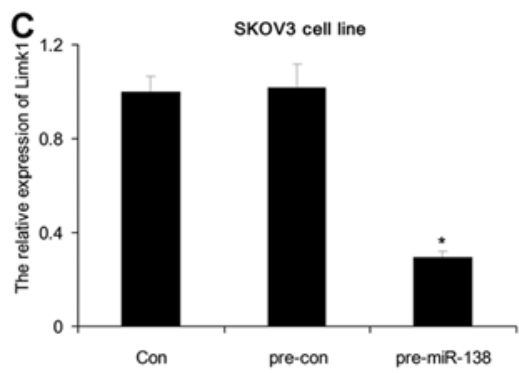
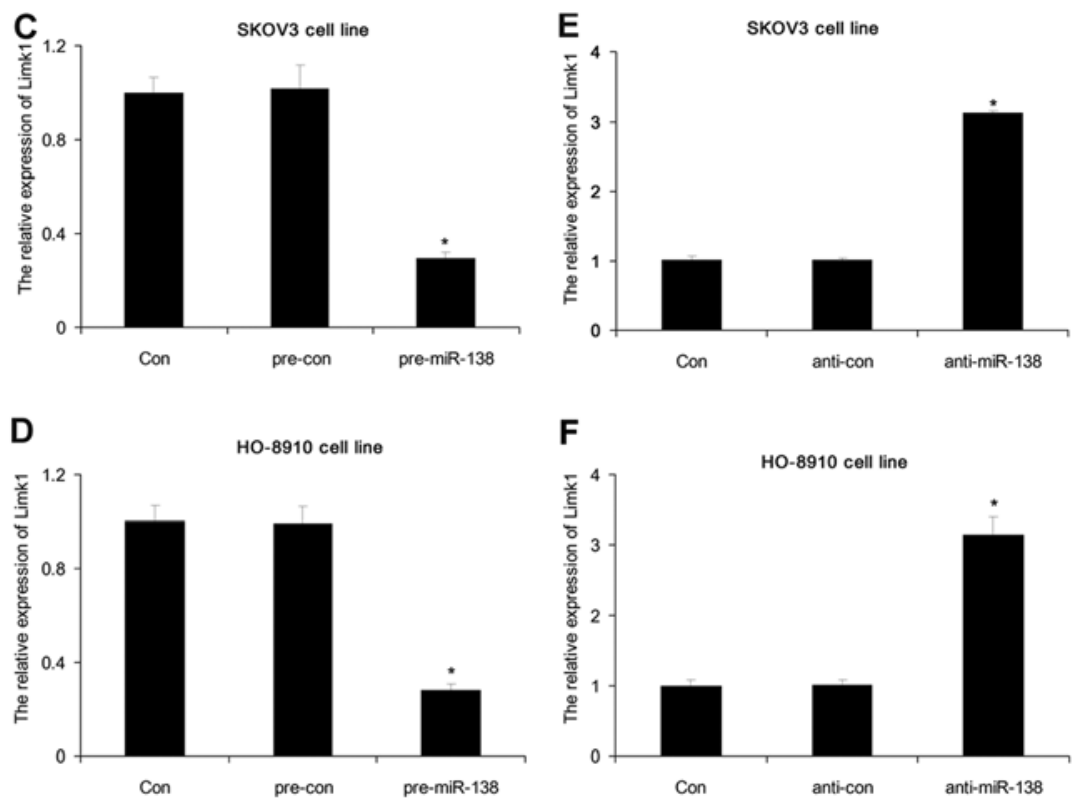

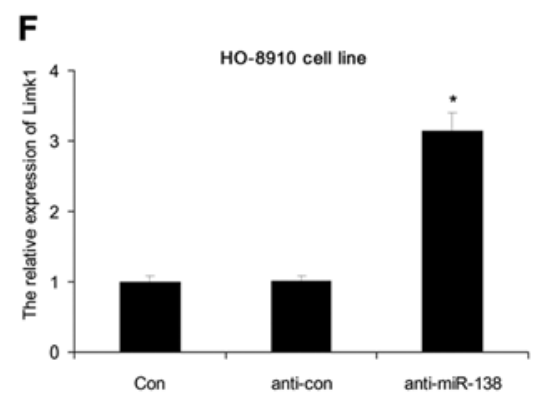

Figure 2. miR-138 targeting Limk1 and the effect on the mRNA level of Limk1. Dual luciferase reporter assays were performed to examine the interaction of miR-138 and its targeting sequence in the Limk1 3'-UTR using constructs containing the targeting sequence (limk1 3'-UTR) and mutated targeting sequence (Mut-Limk1-3'-UTR) in (A) SKOV3 and (B) HO-8910 cells. Overexpression of miR-138 by transfected with pre-miR-138 significantly decreased the Limk1 mRNA level in (C) SKOV3 and (D) HO-8910 cells compared to the control (Con), as detected by RT-qPCR. Downregulation of miR-138 transfected with anti-miR-138 significantly increased the Limk1 mRNA level in (E) SKOV3 and (F) HO-8910 cells compared to the control (Con), as detected by RT-qPCR. ${ }^{*} \mathrm{P}<0.05$ vs. Con, in SKOV3 and HO-8910 cells.

A

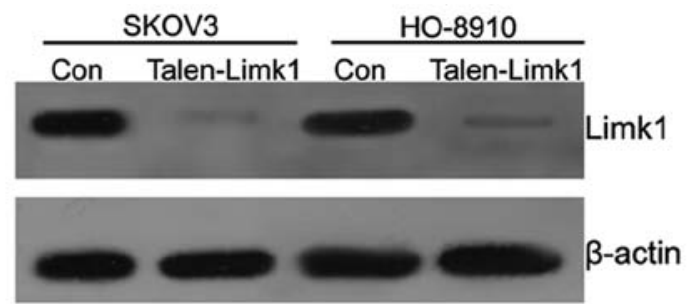

B

SKOV3 cell line

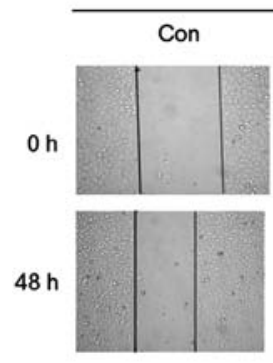

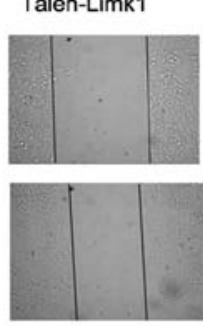

HO-8910 cell line

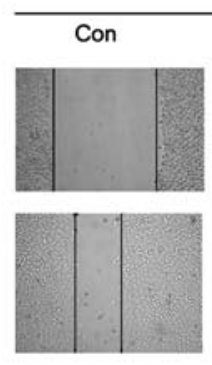

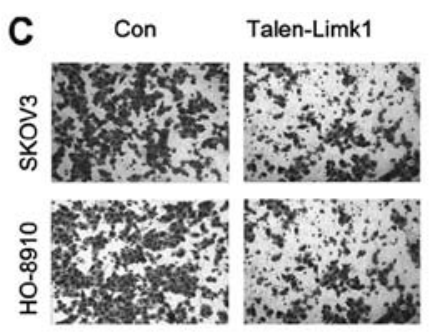

D

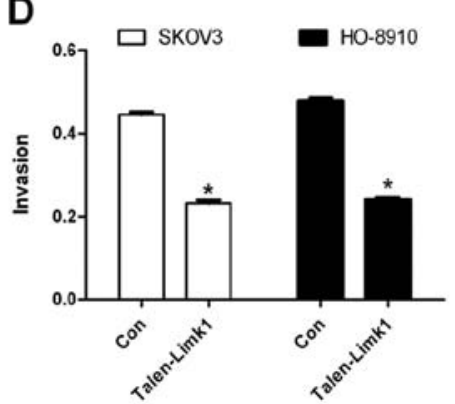

Figure 3. Knockout of Limk1 with Talen technology and the effect on cell invasion and migration. (A) The protein expression of Limk1 after Talen was used to knockout Limk1 in SKOV3 and HO-8910 cells. (B) Cell migratory and (C and D) cell invasive capacity was significantly decreased when Limk1 was knocked out by Talen in SKOV3 and HO-8910 cells compared to the control (Con). "P<0.05 vs. Con, in SKOV3 and HO-8910 cells.

(Fig. 2C and D). However, the inhibition of miR-138 upregulated the mRNA level of Limk1 in both cells (Fig. 2E and F). These findings suggested that the mRNA level of Limk1 was negatively regulated by miR-138 at a post-transcriptional level.

Limk1 promotes invasion and migration of OC cells. To obtain loss-of-function of Limk1, Talen technology was used to construct Limk1 knockout stale cell lines. SKOV3 and HO-8910 cells were transfected with a Talen-Limk1 plasmid pair. The expression of Limk1 in the two cell lines was almost undetectable (Fig. 3A), suggesting that Limk1 was successfully knocked out in the two cell lines. Moreover, it was found that the knockout of Limk1 reduced the cell invasive and migratory abilities of SKOV3 and HO-8910 cells transfected with the Talen-Limk1 plasmid pair (Fig. 3B-D), suggesting that Limk1 promoted the invasion and migration of OC cells. These results suggested that Limk1 plays a promotional role in OC growth and metastasis. 

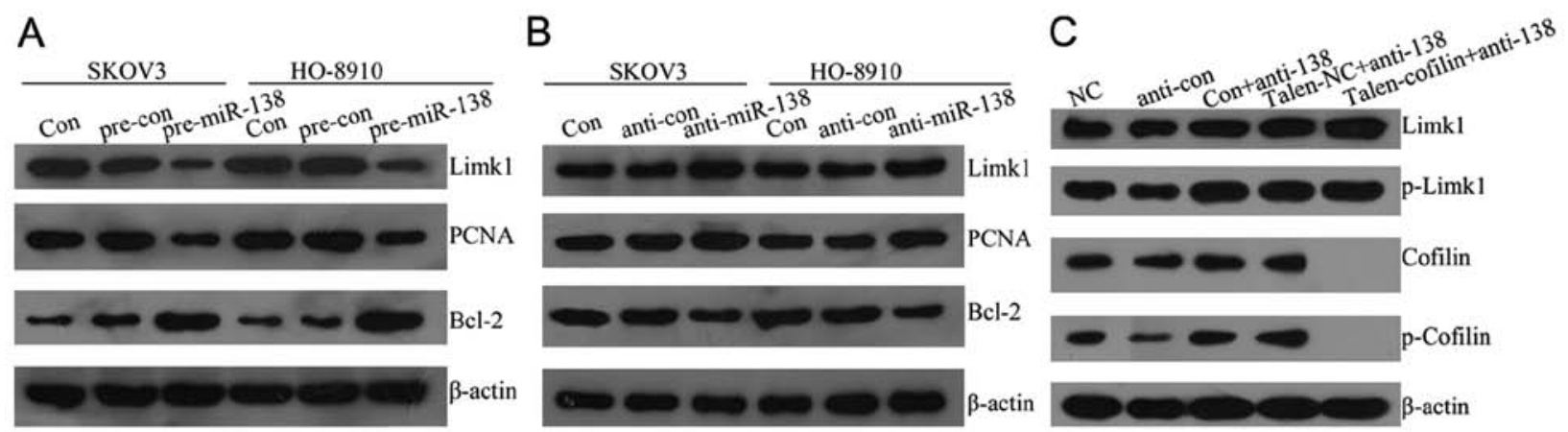

Figure 4. miR-138 targeting Limk1 and its effects on PCNA, Bcl-2 expression and cofilin signaling. Effects of (A) pre-miR-138 and (B) anti-miR-138 on the protein expression of PCNA, Bcl-2 in SKOV3 and HO-8910 cells using western blot analysis. (C) Effects of anti-miR-138 on the cofilin signaling activation in SKOV3 and Talen-cofilin SKOV3 stale cell lines using western blot analysis.

Effects of miR-138 on Limk1/cofilin signaling pathway and the expression of PCNA and Bcl-2. To clarify the underlying molecular mechanisms of miR-138 mediating OC cell viability and metastasis, western blot analysis was performed to detect the proliferation and apoptosis-associated protein. It was found that Limk1 and the proliferation marker PCNA were decreased, whereas the anti-apoptotic marker Bcl-2 was upregulated in SKOV3 and HO-8910 cells transfected with pre-miR-138 (Fig. 4A). Anti-miR-138 increased Limk1 and PCNA expression but decreased Bcl-2 compared to the control (Fig. 4B). The results suggested that miR-138 loss mediated Limk1 upregulation and subsequently promoted PCNA expression and reduced Bcl-2 expression, which plays an important role in cell growth. The activation of cofilin signaling is a classic pathway mediating cell metastasis. To clarify whether miR-138-mediated metastasis was involved in Limk1/cofilin signaling, cofilin was knocked out by Talen technology in the two OC cells to obtain Talen-cofilin stable cell lines. Western blot analysis revealed that anti-miR-138 enhanced the expression levels of Limk1, p-Limk1 and p-cofilin. By contrast, in Talen-cofilin cells, anti-miR-138 was able to increase the expression of Limk1 and p-Limk1 but did not phosphorylate cofilin because of the cofilin knockout (Fig. 4C).

miR-138 suppresses the proliferation, invasion and migration of OC cells. To examine the functional role of miR-138 in OC cells, gain-of-function and loss-of-function cell models were established by transfecting with pre- and anti-miR-138, respectively, in SKOV3 and HO-8910 cell lines. It was confirmed that the overexpression of miR-138 reduced cell viability, while the downregulation of miR-138 increased cell viability in the two OC cells (Fig. 5A and B). Moreover, the invasive and migratory abilities of SKOV3 and HO-8910 cells transfected with pre-miR-138 were reduced. Inversely, cells transfected with anti-miR-138 caused increased cell invasion and migration (Fig. 5C-F). These results suggested that miR-138 served as a suppressor in OC, which was completely opposite to Limk1. Limk1 was also found to be a target of miR-138 in OC cells. Further functional analysis demonstrated that the knockout of cofilin attenuated the anti-miR-138 promotion of invasion and migration of OC cells (Fig. 5C-F), suggesting that miR-138 functioning in $\mathrm{OC}$ is partly involved in activation of the cofilin signaling pathway.

\section{Discussion}

Limk1 is involved in mechanisms regulating cell invasion and may be involved in ovarian carcinoma progression (9). However, the functional role and the underlying molecular mechanism of Limk1 deregulation OC remains to be determined. Results of this study show that the Limk1 was upregulated in OC cells tissues. To examine the functional role of Limk1 in OC cells, we constructed a cell model of Limk1 knockout using Talen. Compared with the control cells, the knockout of Limk1 inhibited the invasion and migration of OC cells, suggesting that Limk1 is an oncogene promoting metastasis in OC. Although Limk1 regulates the growth of some tumor cells $(20,21)$, we observed no significant difference in the proliferation between the two OC cell lines and their corresponding Limk1-Talen cells (data not shown). This finding suggests Limk1 is a cell-specific growth regulator.

miR-138 is a tumor suppressor in different types of cancer cells. It can inhibit cancer cell growth, invasion and migration, and promote apoptosis. In OC, miR-138 also acts as a tumor suppressor by targeting SOX4 (19). Through the prediction of bioformatics software, we found that Limk1 was a theoretic target of miR-138. Fine modulation of Limk1 activity may be a major challenge in the inhibition of tumor cell invasion mediated by one or a combination of the upstream signaling factors. Thus, the downregulation of miR-138 was one of the possible molecular mechanisms of the upregulation of Limk1 and miR-138 inhibited OC progression by targeting Limk1. miR-138/Limk1 may be candidate targets for developing novel therapeutic agents towards OC invasion and metastasis (22). A comparison of cell growth, invasion and migration ability among different cell function models showed that the upregulation of miR-138 (transfected with pre-miR-138) inhibited cell growth, invasion and migration significantly. By contrast, the knockdown of miR-138 (transfected with anti-miR-138) promoted cell growth, invasion and migration significantly. The overexpression of miR-138 was more effective in inhibiting tumors than the Limk1-Talen group, suggesting that Limk1 was not the sole target gene mediating cell growth and metastasis in HO-8910 and SKOV3 cells.

In our functional experiments, we verified miR-138 acted as a tumor suppressor in the SKOV3 and HO-8910 OC cells by inhibiting cell growth, invasion and migration by directly targeting Limk1 via the Limk1/cofilin signaling pathway. To 

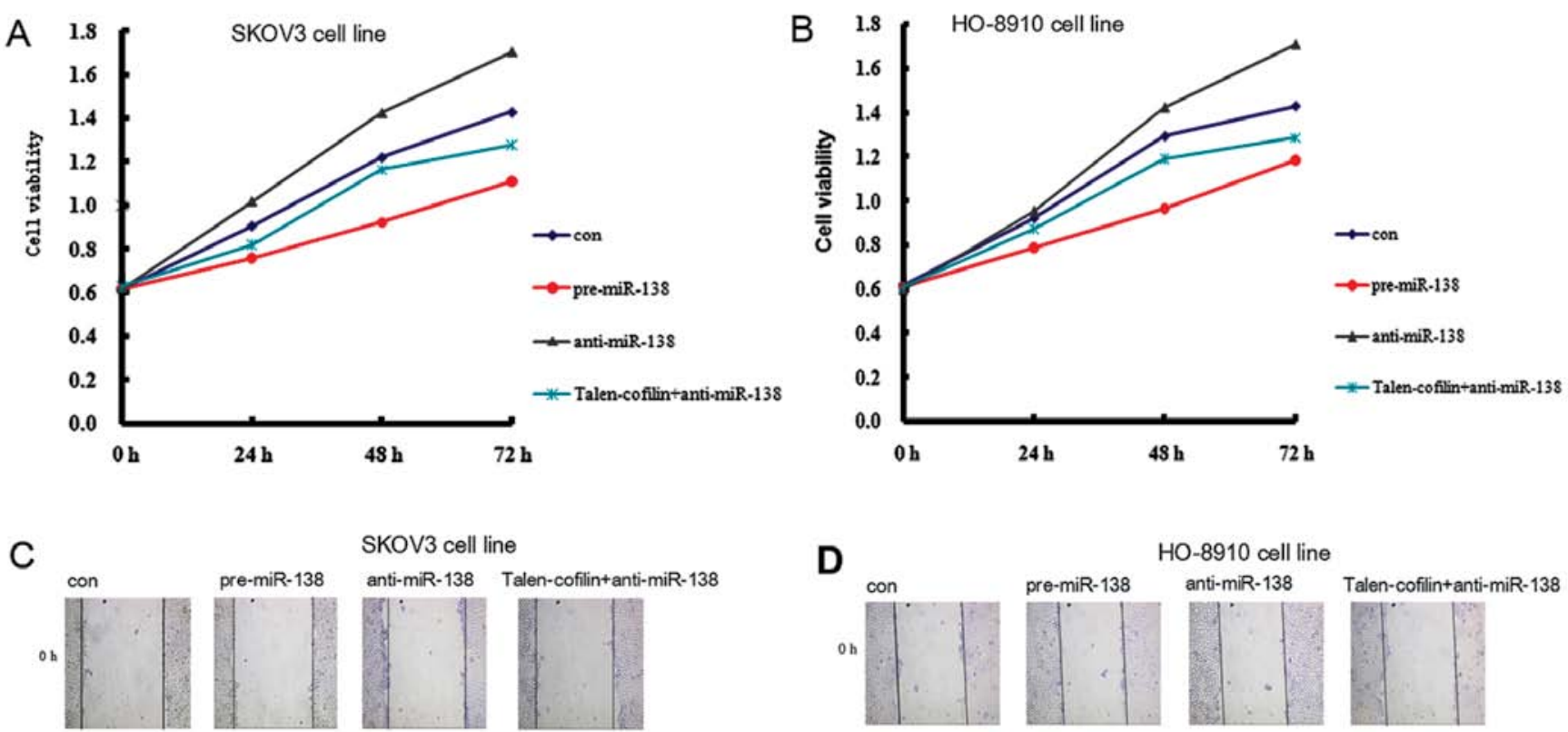

SKOV3 cell line
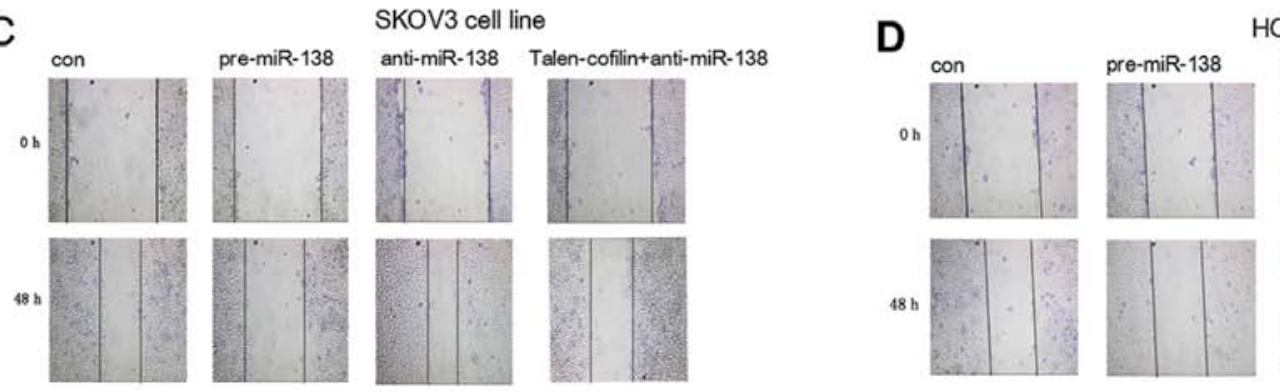

HO-8910 cell line

$\mathrm{E}$
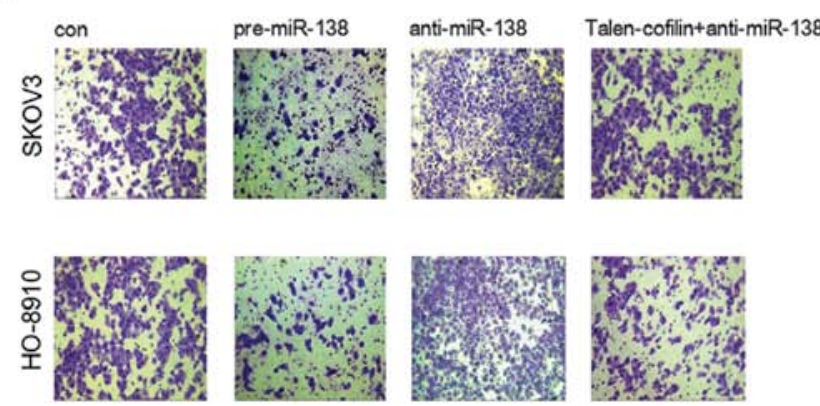

$\mathrm{F}$

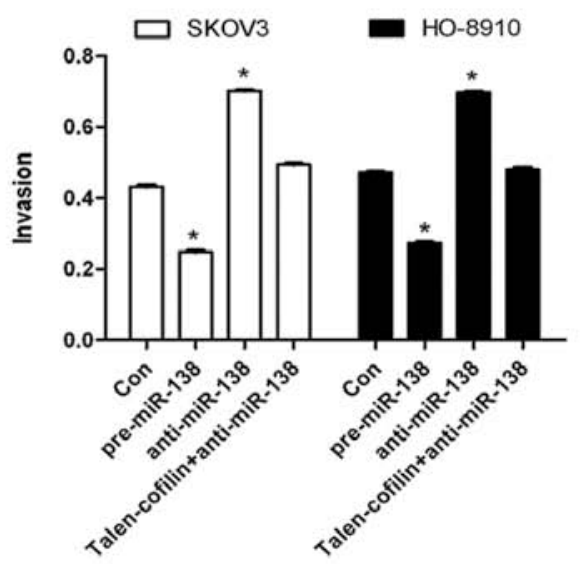

Figure 5. miR-138 suppressed the proliferation, invasion and migration of OC cells via cofilin signaling. The proliferation decreased following transfection with pre-miR-138 and was promoted following transfection with anti-miR-138 in (A) SKOV3 and (B) HO-8910 cells using an MTT assay. However, the promotional effect on the proliferation of anti-miR-138 was reduced in Talen-cofilin stable cell lines. Migration decreased following transfection with pre-miR-138 and was promoted following transfection with anti-miR-138 in (C) SKOV3 and (D) HO-8910 cells using a wound healing assay. However, the promotional effect on the migration of anti-miR-138 was reduced in Talen-cofilin stable cell lines. Cell invasion was decreased following transfection with pre-miR-138 and was promoted following transfection with anti-miR-138 in SKOV3 and HO-8910 (E and F) cells using a Transwell assay. However, the promotional effect on invasion of anti-miR-138 was reduced in Talen-cofilin stable cell lines. ${ }^{~} \mathrm{P}<0.05$ vs. control (Con), in SKOV3 and HO-8910 cells.

verify that Limk1/cofilin signaling participated in regulating the inhibitory effect on OC cells of miR-138, we constructed cofilin knockout stable cell lines using Talen technology. Cofilin is an oncogene whose cell growth, migration and invasion capacity are repressed when cofilin was knocked out by Talen. When we transfected anti-miR-138 in cofilin-Talen stale cell lines, although Limk1 expression was upregulated, the cell growth, migration and invasion ability were not sufficiently promoted. This finding suggests that miR-138 at least partially inhibited cell growth, migration and invasion via the Limk1/ cofilin signaling pathway.

It has been recently reported that Limk1 plays a role in chemosensitivity in non-small cell lung cancer. Limk1 knockdown may sensitize $801 \mathrm{D}$ cells to chemotherapeutic drugs such as cisplatin and gemcitabine (7). miR-138 was found to directly target focal adhesion kinase to increase sensitivity to chemotherapy in cancer cells (23). We aimed to confirm that miR-138 was able to influence chemosensitivity by targeting Limk1 of OC cells. However, we did not obtain results pertaining to knockout of Limk1 or overexpression of miR-138 in our experiments. Concerning SKOV3 cells, it has been reported that the absence of p53 led to its chemoresistance to cisplatin, and whether miR-138/Limk1 regulates chemosensitivity may be associated with different cell types.

In conclusion, the downregulation of miR-138 results in upregulation of its target Limk1 in OC, which promotes cell 
growth and metastasis of OC cells through the Limk1/cofilin signaling pathway. miR-138/Limk1 may therefore be candidate targets for developing novel therapeutic agents towards OC tumor invasion and metastasis.

\section{References}

1. Kigawa J: New strategy for overcoming resistance to chemotherapy of ovarian cancer. Yonago Acta Med 56: 43-50, 2013

2. Miles GD, Seiler M, Rodriguez L, Rajagopal G and Bhanot G: Identifying microRNA/mRNA dysregulations in ovarian cancer. BMC Res Notes 5: 164, 2012.

3. Ozols RF: Treatment goals in ovarian cancer. Int J Gynecol Cancer 15 (Suppl 1): 3-11, 2005.

4. Davila M, Frost AR, Grizzle WE and Chakrabarti R: LIM kinase 1 is essential for the invasive growth of prostate epithelial cells: implications in prostate cancer. J Biol Chem 278 36868-36875, 2003.

5. Zhou Y, Su J, Shi L, Liao Q and Su Q: DADS downregulates the Rac1-ROCK1/PAK1-LIMK1-ADF/cofilin signaling pathway, inhibiting cell migration and invasion. Oncol Rep 29: 605-612, 2013.

6. Nishimura Y, Yoshioka K, Bernard O, Bereczky B and Itoh K: A role of LIM kinase 1/cofilin pathway in regulating endocytic trafficking of EGF receptor in human breast cancer cells. Histochem Cell Biol 126: 627-638, 2006.

7. Chen Q, Jiao D, Hu H, et al: Downregulation of LIMK1 level inhibits migration of lung cancer cells and enhances sensitivity to chemotherapy drugs. Oncol Res 20: 491-498, 2013.

8. Jang I, Jeon BT, Jeong EA, et al: Pak1/LIMK1/Cofilin pathway contributes to tumor migration and invasion in human non-small sell lung sarcinomas and sell lines. Korean J Physiol Pharmacol 16: 159-165, 2012.

9. Zhang W, Gan N and Zhou J: Immunohistochemical investigation of the correlation between LIM kinase 1 expression and development and progression of human ovarian carcinoma. J Int Med Res 40: 1067-1073, 2012.

10. Lin H, Dai T, Xiong H, et al: Unregulated miR-96 induces cell proliferation in human breast cancer by downregulating transcriptional factor FOXO3a. PloS One 5: e15797, 2010.

11. Zhang H, Zhang H, Zhao M, et al: MiR-138 inhibits tumor growth through repression of EZH2 in non-small cell lung cancer. Cell Physiol Biochem 31: 56-65, 2013.
12. Jin $Y$, Chen D, Cabay RJ, Wang A, Crowe DL and Zhou X: Role of microRNA-138 as a potential tumor suppressor in head and neck squamous cell carcinoma. Int Rev Cell Mol Biol 303: 357-385, 2013.

13. Liu X, Jiang L, Wang A, Yu J, Shi F and Zhou X: MicroRNA-138 suppresses invasion and promotes apoptosis in head and neck squamous cell carcinoma cell lines. Cancer Lett 286: 217-222, 2009.

14. Liu X, Wang C, Chen Z, et al: MicroRNA-138 suppresses epithelial-mesenchymal transition in squamous cell carcinoma cell lines. Biochem J 440: 23-31, 2011.

15. Wang W, Zhao LJ, Tan YX, Ren H and Qi ZT: MiR-138 induces cell cycle arrest by targeting cyclin D3 in hepatocellular carcinoma. Carcinogenesis 33: 1113-1120, 2012.

16. Gong H, Song L, Lin C, et al: Downregulation of miR-138 sustains NF-kappaB activation and promotes lipid raft formation in esophageal squamous cell carcinoma. Clin Cancer Res 19: 1083-1093, 2013

17. Long L, Huang G, Zhu H, Guo Y, Liu Y and Huo J: Downregulation of miR-138 promotes colorectal cancer metastasis via directly targeting TWIST2. J Transl Med 11: 275, 2013.

18. Wang Q, Tang H, Yin S and Dong C: Downregulation of microRNA-138 enhances the proliferation, migration and invasion of cholangiocarcinoma cells through the upregulation of RhoC/p-ERK/MMP-2/MMP-9. Oncol Rep 29: 2046-2052, 2013.

19. Yeh YM, Chuang CM, Chao KC and Wang LH: MicroRNA138 suppresses ovarian cancer cell invasion and metastasis by targeting SOX4 and HIF-1alpha. Int J Cancer 133: 867-878, 2013.

20. Wan L, Zhang L, Fan K and Wang J: miR-27b targets LIMK1 to inhibit growth and invasion of NSCLC cells. Mol Cell Biochem 390: 85-91, 2014.

21. Zhang HS, Zhao JW, Wang $\mathrm{H}$, et al: LIM kinase 1 is required for insulin-dependent cell growth of osteosarcoma cell lines. Mol Med Rep 9: 103-108, 2014.

22. Manetti F: Recent findings confirm LIM domain kinases as emerging target candidates for cancer therapy. Curr Cancer Drug Targets 12: 543-560, 2012.

23. Golubovskaya VM, Sumbler B, Ho B, Yemma M and Cance WG: MiR-138 and MiR-135 directly target focal adhesion kinase, inhibit cell invasion, and increase sensitivity to chemotherapy in cancer cells. Anticancer Agents Med Chem 14: 18-28, 2014. 